\title{
Editorial: General Relativity Still Making Waves
}

This year marks the 100th anniversary of Einstein's general theory of relativity. In a series of papers published in November 1915 [1] and May 1916 [2], Einstein upended the Newtonian concept of gravitation as a force acting instantaneously over a great distance, and replaced it with the idea that gravity is "geometry," a consequence of the curvature or warpage of space and time. Einstein's theory has completely transformed our view of space, time, and the Universe. But it was only the beginning of a story that physicists are still writing.

Einstein and his general theory became celebrities in November 1919, when newspapers worldwide proclaimed "Einstein's theory triumphs." The occasion was the report by British astronomers that they had measured the bending of starlight by the Sun. The researchers had analyzed photographs of stars near the Sun during a total eclipse and found that the tiny displacements of their images, with respect to reference photographs, agreed better with Einstein's theory than with Newton's, which predicted half the effect [3]. Subsequent eclipse measurements tended to confirm Einstein, but some physicists and astronomers remained skeptical [4]. While Einstein's theory successfully accounted for an anomaly in the orbit of Mercury, a third test of the theory, called the gravitational redshift, was initially a bust. Two 1917 observations failed to detect the predicted shift in the Sun's spectral lines. It wasn't until 1960 that the effect was finally measured in a laboratory experiment involving gamma rays [5].

The difficulty in testing the theory led many to think its effects were too small to be relevant, and, during the 1920s and 30s, interest in the theory declined. By 1960 it was relegated to the backwaters of physics and astronomy. The discovery of quasars in the early 1960s changed all this. The unprecedented outpouring of energy from these enigmatic objects required a powerful source, and some scientists turned to the relativistic black hole, the ultimate spacetime warper, for a solution. Technological advances and the advent of space exploration provided new tools that could properly test general relativity. Thus began a general relativity renaissance [6].

Today general relativity is at the forefront of physics and astronomy $[7,8]$, and may be key to answering the big questions of our time: What is the origin of our Universe and what will its fate be? What does gravity look like on quantum scales? What are black holes? General relativity is now "big" science, involving kilometer-scale observatories designed to detect gravitational waves [9], and the world's largest supercomputers are simulating the mergers of black holes [10]. Ideas from general relativity are feeding back into other fields like condensed matter and nuclear physics, which use Einstein's mathematics to solve otherwise intractable problems [11]. The theory has found its way into daily life: relativistic effects must be accounted for if global positioning systems are to work properly $[12,13]$. It is even seeping into popular culture with general relativity featuring in Hollywood movies like Interstellar and The Theory of Everything.

The next century of general relativity looks to be as exciting as the first. The anticipated detection of gravitational waves should yield further confirmation of Einstein's theory, and provide new ways of observing the Universe. More detailed studies of black holes may uncover how they form, grow, and whether their strong warping of space-time is as Einstein predicted. And theorists will continue to seek a formulation of gravity - possibly different from Einstein's 1915 version - that achieves the long-sought quantum theory of gravity.

\author{
Clifford Will \\ Department of Physics, \\ University of Florida, \\ P.O. Box 118440, Gainesville, \\ Florida 32611-8440, USA
}

Published 24 September 2015

DOI: 10.1103/PhysRevD.92.060001

PACS numbers: $01.30 . \mathrm{Ww}$ 
[1] A. Einstein, Sitzungsberichte der Königlich Preussischen Akademie der Wissenschaften, pp. 778-786, 799-801, 831-839, 844-847 (Berlin, 1915).

[2] A. Einstein, Die Grundlage der allgemeinen Relativitätstheorie, Ann. Phys. (Berlin) 354, 769 (1916).

[3] C. M. Will, The 1919 measurement of the deflection of light, Classical Quantum Gravity 32, 124001 (2015).

[4] J. Crelinsten, Einstein's Jury: The Race to Test Relativity (Princeton University Press, Princeton, NJ, 2006).

[5] R. V. Pound and G. A. Rebka, Apparent Weight of Photons, Phys. Rev. Lett. 4, 337 (1960).

[6] C. M. Will, Was Einstein Right? Putting General Relativity to the Test (Basic Books, New York, 1993).

[7] General Relativity and Gravitation: A Centennial Perspective, edited by A. Ashtekar et al., (Cambridge University Press, Cambridge, England, 2015).
[8] C. M. Will, Focus issue: Milestones of general relativity, Classical Quantum Gravity 32, 124001 (2015).

[9] R. X. Adhikari, Gravitational radiation detection with laser interferometry, Rev. Mod. Phys. 86, 121 (2014).

[10] J. Centrella, J. G. Baker, B. J. Kelly, and J. R. van Meter, Black-hole binaries, gravitational waves, and numerical relativity, Rev. Mod. Phys. 82, 3069 (2010).

[11] S. A. Hartnoll, Lectures on holographic methods for condensed matter physics, Classical Quantum Gravity 26, 224002 (2009).

[12] C. M. Will, Einstein's Relativity and Everyday Life, Physics Central Writers' Gallery, http://physicscentral.com/explore/ writers/will.cfm.

[13] N. Ashby, Relativity and the global positioning system, Phys. Today 55, 41 (2002). 